\title{
The Enhancement of Energy Storage Performances via Combining Relaxor Behaviors With the Crucial Point of Solubility Limit
}

\section{Zhifeng Shi}

Inner Mongolia University

Yaping Liu

Inner Mongolia University

Fei Guo

Inner Mongolia University

Shifeng Zhao ( $\nabla$ zhsf@imu.edu.cn )

Inner Mongolia University https://orcid.org/0000-0002-3702-9144

\section{Research Article}

Keywords: relaxor, solubility limit, energy storage, local component fluctuation

Posted Date: February 15th, 2021

DOI: https://doi.org/10.21203/rs.3.rs-193213/v1

License: (9) This work is licensed under a Creative Commons Attribution 4.0 International License.

Read Full License

Version of Record: A version of this preprint was published at Journal of Materials Science: Materials in Electronics on April 16th, 2021. See the published version at https://doi.org/10.1007/s10854-021-058938. 


\section{Abstract}

$(1-\mathrm{x})\left(0.96 \mathrm{Na}_{0.5} \mathrm{Bi}_{0.5} \mathrm{TiO}_{3}-0.04 \mathrm{BiMnO}_{3}\right)-\mathrm{xLaMnO}$ ternary solid solution films were fabricated by Sol-gel methods. It takes advantage of the large polarization from 0.96NBT-0.04BMO limit solid solution at crucial point of solubility limit to enhance energy density. And $\mathrm{LaMnO}_{3}$ was introduced to improve the energy storage efficiency via the enhancement of relaxor behaviors. It is shown that the relaxor behaviors had been enhanced, and a large energy storage density of $87.9 \mathrm{~J} / \mathrm{cm}^{3}$ and efficiency of $56.5 \%$ were achieved for 0.85 (NBT-BMO)-0.15LM films, with increase ratio of $4.9 \%$ and $13.3 \%$ respectively than 0.96NBT-0.04BMO solubility limit films. However, when $\mathrm{LaMnO}_{3}$ is beyond a certain limit, the breakdown strength is reduced due the formation of the current channel. Thus it suggests a alternative method that combining relaxor behaviors with solubility limit films, which provides a way to regulate the energy storage performances for film capacitors.

\section{Introduction}

How to effectively store energy, reduce energy consumption and weaken environmental burden has become a hot issue for the researchers in recent years. ${ }^{[1-3]}$ Most renewable energy is converted into electricity. Efficient storage technologies are developed such as batteries, super capacitors, and dielectric capacitors. The batteries endow the highest energy storage density and low power density. The super capacitors have medium energy storage density and power density, however, the complex structure, huge environmental pollution and short cycle limit their application. ${ }^{[4]}$ Interestingly, although the dielectric capacitors have low energy storage density $(<30 \mathrm{w} \cdot \mathrm{h} / \mathrm{kg}$ ), the advantages of high power $(108 \mathrm{~W} / \mathrm{kg})$, wide temperature range and long cycle time are beneficial to rapid charge and discharge of the devices such as electronic system and pulsed laser, which have been arousing enormous interest. ${ }^{[5-7]}$

Therefore, the researchers devote several efforts to enhance the energy density of dielectric capacitors via preparing solid solution, domain engineering and core-shell structure. ${ }^{[8-10]}$ On one hand, the insulation characteristics are improved by forming multi-layer interface, producing charge accumulation. On the other hand, the polarization states are regulated by changing the internal structure including modifying the lattices and the domains. Among them, limit solid solution materials intensify the off-center position and polarization by large lattice distortion, with polarization enhancement of $53.8 \%$, which is so effective that it improves the energy storage density. ${ }^{[11]}$ However, they endow low energy storage efficiency due to the weaken linear behaviors. For dielectric film capacitors, the discharge energy storage density $\left(U_{r}\right)$ and efficiency $(\eta)$ are calculated based on the ferroelectric hysteresis loops, ${ }^{[12]}$

$$
\begin{aligned}
& U_{\mathrm{s}}=\int_{P_{\mathrm{s}}}^{P_{\mathrm{m}}} E \mathrm{~d} P \\
& \eta=\frac{U_{\mathrm{s}}}{U}=\frac{U_{\mathrm{r}}}{U_{\mathrm{r}}+U_{1005}} \times 100 \%
\end{aligned}
$$


where $E_{,} P_{\mathrm{m}}, P_{\mathrm{r}}$, and $U_{\text {loss }}$ are the electric field strength, maximum polarization, residual polarization, and the energy loss, respectively. Thus, reducing the energy dissipation is a key factor to enhance the energy storage efficiency for limit solid solution films. Relaxor ferroelectrics have the advantages of low energy dissipation and large breakdown field strength, which is attributed to the disorder of the internal structure and nano polarization regions. ${ }^{[13,14]}$ Thus, combining the large polarization of limit solid solutions with low energy dissipation of relaxor behaviors is expected to become an effective way to achieve excellent energy storage performances.

Therefore, this work designed (1- $\mathrm{x})\left(0.96 \mathrm{Na}_{0.5} \mathrm{Bi}_{0.5} \mathrm{TiO}_{3}-0.04 \mathrm{BiMnO}_{3}\right)-\mathrm{xLaMnO}{ }_{3}(\mathrm{x}=0.10,0.15$ and 0.20$)$ (NBT-BMO-LM) ternary solid solution films. On the one hand, it takes the advantage of the large polarization of NBT-BMO limit solid solution films at crucial point of solubility limit. On the other hand, LM was introduced to improve the efficiency via relaxor behaviors. When more LM were introduced, the relaxor behaviors are enhanced, however, when LM is beyond a certain limit, the breakdown strength is decreased due to the formation of the current channel. Noticeably, the energy density of $87.9 \mathrm{~J} / \mathrm{cm}^{3}$ and efficiency of $56.5 \%$ were achieved in 0.85 (NBT-BMO)-0.15LM films. The mechanism of combining relaxor behaviors with the crucial point of solubility limit is detailedly discussed.

NBT-BMO-LM solid solution films were prepared by a cast-effective chemical solution deposition. $\mathrm{Mn}(\mathrm{CH} 3 \mathrm{COO})_{3} \cdot \mathrm{xH}_{2} \mathrm{O}(96 \%)$ and $\mathrm{Bi}\left(\mathrm{NO}_{3}\right)_{3} \cdot 5 \mathrm{H}_{2} \mathrm{O}(99.0 \%)$ were dissolved in 2-methoxyethannol to form a precursor solution $\mathrm{A}$. $\mathrm{Bi}\left(\mathrm{NO}_{3}\right)_{3} \cdot 5 \mathrm{H}_{2} \mathrm{O}(99.0 \%)$, $\left.\mathrm{La}(\mathrm{NO} 3)_{3} \cdot 6 \mathrm{H}_{2} \mathrm{O}\right)(99.99 \%)$ and $\mathrm{CH}_{3} \mathrm{COONa}(99.0 \%)$ were dissolved in ethylene glycol to form a precursor solution B. Solution $\mathrm{A}$ and $\left.\mathrm{CH}_{3}\left(\mathrm{CH}_{2}\right)_{3} \mathrm{O}\right]_{4} \mathrm{Ti}(98.0 \%)$ were dripped into solution $\mathrm{B}$ to form a clear precursor solution. The precursor solutions were deposited onto $\mathrm{Pt} / \mathrm{Ti} / \mathrm{SiO}_{2} / \mathrm{Si}(100)$ square substrate with dried temperature and annealing temperature of $350^{\circ} \mathrm{C}$ and $620^{\circ} \mathrm{C}$, respectively. And repeat above steps until the thickness of the films is $500 \mathrm{~nm}$. The crystal structures was measured in X-ray diffractometer (XRD, Panalytical Empyrean). The measurement of the ferroelectric and leakage properties were carried out by a radiant multiferroic tester system (RT-500). The temperature-dependent dielectric permittivity and loss are characterized in impedance analyzer (Agilent E4990A) with variable-temperature probe station.

Fig. 1 (a) shows the XRD patterns of (1-x)(0.96NBT-0.94BMO)-x $\mathrm{LaMnO}_{3}(\mathrm{x}=0.10,0.15$ and 0.20$)$ ternary solid solution films. The diffraction peaks are marked by the pseudo-cubic $\mathrm{Na}_{0.5} \mathrm{Bi}_{0.5} \mathrm{TiO}_{3}$ (ICS Card No. 192002) with Pm3m space group. It is obvious that $L M$ has entered into the lattices without impurity phases except for substrate peaks and the solvent atoms peaks of BM, which indicates NBT-BM-LM limit solid solution is at the crucial point of solubility limit. ${ }^{[11]}$ The detailed enlarged view has been displayed in

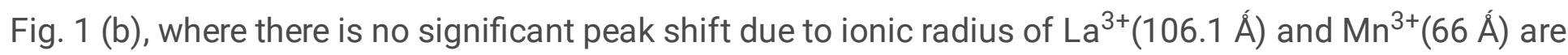
slightly different from $\mathrm{Bi}^{3+}(108 \AA \hat{)})$ and $\mathrm{Ti}^{4+}(68 \AA \hat{A})$ respectively. And the precipitation of $\mathrm{BM}$ is indexed by the monoclinic phase BiMnO3 (ICS Card No. 245299). Noticeably, the relative peak intensity of (110) gradually declines with the increase of LM, which reveals that the crystal orientation tends to be in disorder due to the local composition fluctuation. 
Fig. 2 (a-c) displays the ferroelectric loops of NBT-BM-LMO solid solution films. As shown in different proportions, the $P$-E loops display standard and pinched ferroelectric hysteresis loops under near their maximum electric fields of $2970 \mathrm{kV} / \mathrm{cm}, 3566 \mathrm{kV} / \mathrm{cm}$ and $2774 \mathrm{kV} / \mathrm{cm}$ respectively. It is obvious that the large maximum polarization, the lower remanent polarization and smaller coercive fields are obtained in NBT-BM-LM ternary solid solution films, which is beneficial to energy storage. As a contrast, the $P-E$ loops at $2731 \mathrm{kV} / \mathrm{cm}$ under different LM proportions have been shown in Fig. 2 (d). It is evident for the maximum polarization to decline with the increasing of $L M$. The introduction of solute atom intensifies the local component disorder, which breaks the long-range orders and macroscopic domain, forming the nano polarization regions. The nano-domain depresses the polarization because it needs larger electric field to drive dipoles reorientation along the electric field. Thus, with the increase in the density of the nano polarization regions, the relaxor behaviors have been enhanced.

To further investigate the relaxor behaviors, the temperature-dependent dielectric constant and loss for 0.9(NBT-BMO)-0.1LM, 0.85(NBT-BMO)-0.15LM and 0.8(NBT-BMO)-0.2LM ternary solid solution films had been measured, as shown in Fig. 3 (a-c), respectively. Fig. 3 (a) shows the obvious dielectric dispersion peaks at $278{ }^{\circ} \mathrm{C}$, and the phase change is a gradual process instead of abrupt transformation, which is attributed to the multiply phase structure resulted from the local composition fluctuation. With the increase of LM proportion, Fig. 3 (b) shows a peak at $260^{\circ} \mathrm{C}$, where the dispersion phase transition is strengthened, and obvious chromatic dispersion phenomenon that the peak moves toward high temperature with the increase of frequency, which means the enhancement of the local composition fluctuation. Continue to increase LM proportion, there is no significant dielectric peaks were obtained, as shown in Fig. 3 (c), and the peaks tend to more flat around $253^{\circ} \mathrm{C}$, which means the intense dispersion phase transition resulted from the great local composition fluctuation. Fig. 3 (d) displays the dispersion coefficient $y$ of dielectric constant curves under LM proportions of $0.1,0.15$ and 0.2 , and the corresponding $y$ are 1.54, 1.62 and 1.72 respectively. The values gradually increase when the more LM solutes enters into lattice, which results larger component fluctuation. And the dispersion coefficient tends to 2 , which means the transition from the typical ferroelectrics to relaxor ones. ${ }^{[15]}$

The leakage current characteristics of the solid solution films are shown in Fig. 4 (a). As can be seen, the ternary solid solution films shows well leakage characteristics with leakage density lower than $10^{-3} \mathrm{~J} / \mathrm{cm}^{2}$ at the high electric field of $1545 \mathrm{kV} / \mathrm{cm}$. To gain more insights, the enlarged view has been shown in the inset of Fig. 4 (a), where the leakage current slightly declines with the LM proportion increasing from 0.10 to 0.15 . It is mainly attributed to the fact that the larger component fluctuation leads to the high level local component and the difference of the structure induces the interfacial effect, which scatterings the free electrons and inhibits the current formation. ${ }^{[16]}$ However, the leakage current tends to rise when LM proportion is 0.2 , which is ascribed to the more introduced scattering components, which increases the scattering probability beyond a certain range, thus makes it easier to form the current channels.

The leakage characteristics have a obvious effect on the breakdown field. Weibull distribution is carried out to demonstrate the breakdown field strengths of the ternary solid solution films, which is expressed 
by ${ }^{[17]}$

$$
P(E)=1-\exp \left[-\left(E / E_{D B S}\right)^{\beta}\right]
$$

where $E$ is the measured electric field, $E_{\mathrm{DBS}}$ is the breakdown field strength, $\beta$ is the linear dispersion constant, and $P(E)$ is the cumulative probability of the breakdown. Fig. 4 (b) shows the results of Weibull distributions, and it is obvious that the breakdown electric field increases first and then decreases with the increase of LM proportions. The tendency is similar to that of the leakage characteristics, and the fact is that accumulation of electrons is a mainly way to produce the breakdown for dielectric capacitors. The rapid accumulation of electrons derived from the excited outer-shell electrons exceeding the potential barrier and injected electrons from the electrodes would generate the current channel in order to form the electric tree. ${ }^{[18]}$ Thus, the leakage current characteristics always reveal the change tendency of the breakdown field strength. Interestingly, the breakdown electric fields are $3078 \mathrm{kV} / \mathrm{cm}, 3689 \mathrm{kV} / \mathrm{cm}$ and $2890 \mathrm{kV} / \mathrm{cm}$ for the 0.9(NBT-BMO)-0.1LM, 0.85(NBT-BMO)-0.15LM and 0.8(NBT-BMO)-0.2LM solid solution films respectively, which indicates the great dielectric endurance characteristics.

Fig. 5 (a) displays the energy densities of the ternary solid solution films calculated from the ferroelectric loops. The energy densities reach $74.8 \mathrm{~J} / \mathrm{cm}^{3}, 87.9 \mathrm{~J} / \mathrm{cm}^{3}$ and $67.5 \mathrm{~J} / \mathrm{cm}^{3}$ for 0.9 (NBT-BMO)-0.1LM, 0.85 (NBT-BMO)-0.15LM and 0.8(NBT-BMO)-0.2LM at their maximum electric fields respectively, where the energy densities increase first and then drop. The maximum energy density is obtained for 0.85 (NBTBMO)-0.15LM solid solution films due to the improved relaxor behaviors and the breakdown field strength. However, more introduction of solute LM atoms, high leakage current density depresses the breakdown field, which results in decrease of energy density for 0.8(NBT-BMO)-0.2LM films. Noticeably, the energy density has been increased by $4.9 \%$ from 0.96 NBT-0.04BMO solubility limit film with the energy density of $83.8 \mathrm{~J} / \mathrm{cm}^{3}$. Moreover, the energy densities decrease with the increase in the LM proportions at the same electric fields, and it is attributed to the enhanced relaxor behaviors and the dropped polarization response. Corresponding energy storage efficiency have been shown in Fig. 5 (b). And the efficiency of 0.85 (NBT-BMO)-0.15LM is $58.6 \%$ at the maximum electric fields. Compared with the 0.96NBT-0.04BMO solubility limit films, the energy storage efficiency increases by $13.3 \%$ for 0.85 (NBTBMO)-0.15LM solid solution films. It indicates the introduction of relaxor behaviors via enhancing local component fluctuation is an effective method to improve efficiency. Thus the energy density of 87.9 $\mathrm{J} / \mathrm{cm}^{3}$ with efficiency of $58.6 \%$ has been achieved for the 0.85 (NBT-BMO)- $0.15 \mathrm{LM}$ ternary solid solution films.

Fig. 5 (c) shows the thermal stability of the ternary solid solution films from room temperature to $120^{\circ} \mathrm{C}$. When the temperature increases, the energy storage density is almost unchanged with energy density of 0.85 (NBT-BMO)-0.15LM films slightly decreasing from $44.3 \mathrm{~J} / \mathrm{cm}^{3}$ to $42.6 \mathrm{~J} / \mathrm{cm}^{3}$, which just decreasing by $3.8 \%$. Moreover, the energy storage efficiency of ternary solid solution films also has a weakened impact by temperature. The efficiency of 0.85(NBT-BM0)-0.15LM films reduces from $73.1 \%$ to $65 \%$, which just decreases by $11.1 \%$. Interestingly, the decrease rate gradually increases with the increase in 
LM proportions, which is attributed to the fact that relaxor response time is shorten by the higher temperature, which accelerates the vibration of dipoles. ${ }^{[19]}$ Thus, the temperature has a larger impact on the materials with higher relaxor behaviors. Fig. 5 (d) shows the frequency stability of ternary solid solution films from the frequency of $10 \mathrm{kHz}$ to $33 \mathrm{kHz}$. As shown in Fig. 5 (d), the energy storage density slightly decreases with the increase of frequency under different proportions, which is ascribed to the fact that the switch of the dipoles is so slow that they cannot keep up with the applied external electric field. For 0.85 (NBT-BMO)-0.15LM films, the energy density decreases from $39.1 \mathrm{~J} / \mathrm{cm}^{3}$ to $35.9 \mathrm{~J} / \mathrm{cm}^{3}$, which just decrease by $8.2 \%$. Besides, the energy storage efficiency with different LM proportions gradually increases with the increase in frequency because the incomplete switch of the dipoles reduces the frictional and thermal loss during the polarization process. Therefore, the ternary solid solution films endow the excellent stability of temperature and frequency, which can be used in harsh environment.

In summary, the combining relaxor behaviors with solubility limit films is an effective mean to enhance the energy density and improve the energy storage efficiency. 0.85(NBT-BMO)-0.15LM ternary solid solution films show a large energy density of $87.9 \mathrm{~J} / \mathrm{cm}^{3}$ with efficiency of $58.6 \%$, with increase ratio of $4.9 \%$ and $13.3 \%$ respectively than $0.96 \mathrm{NBT}-0.04 \mathrm{BMO}$ solubility limit films. And the relaxor behaviors of ternary solid solution films improve the leakage current characteristics and the breakdown strength due to the great local component fluctuation. However, the introduction of LM cannot prevent the formation of the current channel from breakdown beyond a certain range. Thus, it indicates a design method that the combining relaxor behaviors with solubility limit to improve the energy storage performances.

This work was financially supported by the National Natural Science Foundation of China (Grant Nos. 12074204, 11864028).

The authors declare that they have no known competing financial interests or personal relationships that could have appeared to influence the work reported in this paper.

\section{References}

1. Chu, X. Zhou, K. Ren, B. Neese, M. Lin, Q. Wang, F. Bauer, Q. M. Zhang, A Dielectric polymer with High Electric Energy Density and Fast Discharge Speed, Science, 313, 334(2006)

2. Prateek, V. K. Thakur, R. K. Gupta, Recent Progress on Ferrelectric polymer-Based Nanocomposites for High Energy Density Capacitors:Synthesis, Dielectric Properties, and Future Aspects, Chem. Rev, 116, 4260(2016)

3. Li, L. Chen, M. R. Gadinski, S. Zhang, G. Zhang, H. Li, A. Haque, L. Q. Chen, T. Jackson, Q. Wang, Flexible high-temperature dielectric matericals form polymer nanocomposites, Nature, 523, 276(2015)

4. Hao, A Review on the dielectric materials for high energy-storage application, Journal of Advanced Dielectrics, 3, 1330001(2013) 
5. X. Zhang, Y. Shen, B. Xu, H. Zhang, L. Gu, J. Y. Jiang, J. Ma, Y. H. Lin, C. W. Nan, Giant Energy Density and Improved Discharge Efficiency of solution-Processd Polymer Nanocomposites for Dielectric Energy Storage, Adv. Mater, 28 2055(2016)

6. B. Pan, L. M. Yao, J. W. Zhai, X. Yao, H. Chen, Interfacial coupling effect in organic/inorganic nanocomposites with high energy density, Adv. Mater, 30, 1705662 (2018)

7. L. Fan, C. R. Ma, Y. Li, Z. S. Liang, S. Cheng, M. Y. Guo, Y. Z. Dai, C. S. Ma, L. Lu, W. Wang, L. H. Wang, X. J. Lou, M. Liu, H. Wang, C. L. Jia, Realization of high energy density in an ultra-wide temperature range though engineering of ferroelectric sandwich structures, Nano Energy, 62, 725 (2019).

8. Guo, Z. F. Shi, B. Yang, Y. P. Liu, S. F. Zhao, Flexible lead-free $\mathrm{Na}_{0.5} \mathrm{Bi}_{0.5} \mathrm{TiO}_{3}-\mathrm{EuTiO}_{3}$ soild solution film capacitors with stable energy storage performances, Scripta Materialia, 184, 52(2020)

9. Pan, J. Ma, Q. H. Zhang, X. Z. Liu, B. Guang, L. Gu, X. Zhang, Y. J. Zhang, L. L. Li, Y. Shen, Y. H. Lin, C. W. Nan, Giant energy density and high efficiency achieved in bismuth ferrite-based film capacitors via domain engineering, Nature Communications, 9, 1813(2018)

10. Guo, Z. F. Shi, B. Yang, S. F. Zhao, The role of PN-like junction effects in energy storage performances for $\mathrm{Ag}_{2} \mathrm{O}$ nanoparticle dispersed lead-free $\mathrm{K}_{0.5} \mathrm{Na}_{0.5} \mathrm{NbO}_{3}-\mathrm{BiMnO}_{3}$ films, Nanoscale, 12. 7544(2020)

11. Guo, Z. F. Shi, Y. P. Liu, S. F. Zhao, Energy storage performances regulated $\mathrm{BiMnO}_{3}$ proportion in limited solid solution films, Chinese Phys. B, 11, 116801(2020).

12. Yao, Z. Song, H. Hao, Z. Yu, M. Cao, S. Zhang, M. Lanagan, H. Liu, Homogeneous/Inhomogeneousstruvtured Dielectric and their Energy-Storage Performances, Adv. Mater, 29, 1601727(2017).

13. Schaab, T. Granzow, Temperature dependent switching machanism of $\left(\mathrm{Pb}_{0.92} \mathrm{La}_{0.08}\right)$ $\left(\mathrm{Zr}_{0.65} \mathrm{Ti}_{0.35}\right) \mathrm{O}_{3}$ investigated by small and large signal measurements, Appl. Phys. Lett, 97, 132902(2010)

14. Y. Chen, Z. H. Tang, B. Yang, S. F. Zhao, High energy storage performances in lead-free $\mathrm{BaBi}_{3.9} \mathrm{Pr}_{0.1} \mathrm{Ti}_{4} \mathrm{O}_{15}$ relaxor ferroelectric films, Appl. Phys. Lett, 15, 153904(2018)

15. Cui, Y. D. Hou, S. Wang, C. Wang, M. K. Zhu, Relaxor behavior of (Ba,Bi)(Ti,Al)O3 ferrelectric ceramic, J. Appl. Phys, 107, 054105(2010)

16. Guo, N. Jiang, B. Yang, S. F. Zhao, Segregation particles induced ultrahigh energy storage performances in $\mathrm{BiMnO}_{3}$ modified $\mathrm{BaTiO}_{3}$ films, Appl. Phys. Lett, 113, 253901(2019)

17. A. Kishimoto, K. Koumoto, H. Yanagida, Mechanical and dielectric failure of BaTiO3 ceramics, Mater. Sci, 24 698(1989)

18. Y. F. Wang, J. Cui, Q. B. Yuan, Y. J. Niu, Y. Y. Bai, H. Wang, Significantly enhanced breakdown strength and energy density in sandwich-structured barium titanate/poly(vinylidene fluoride) nanocomposites, Adv, Mater, 27, 6658(2015)

\section{Figures}




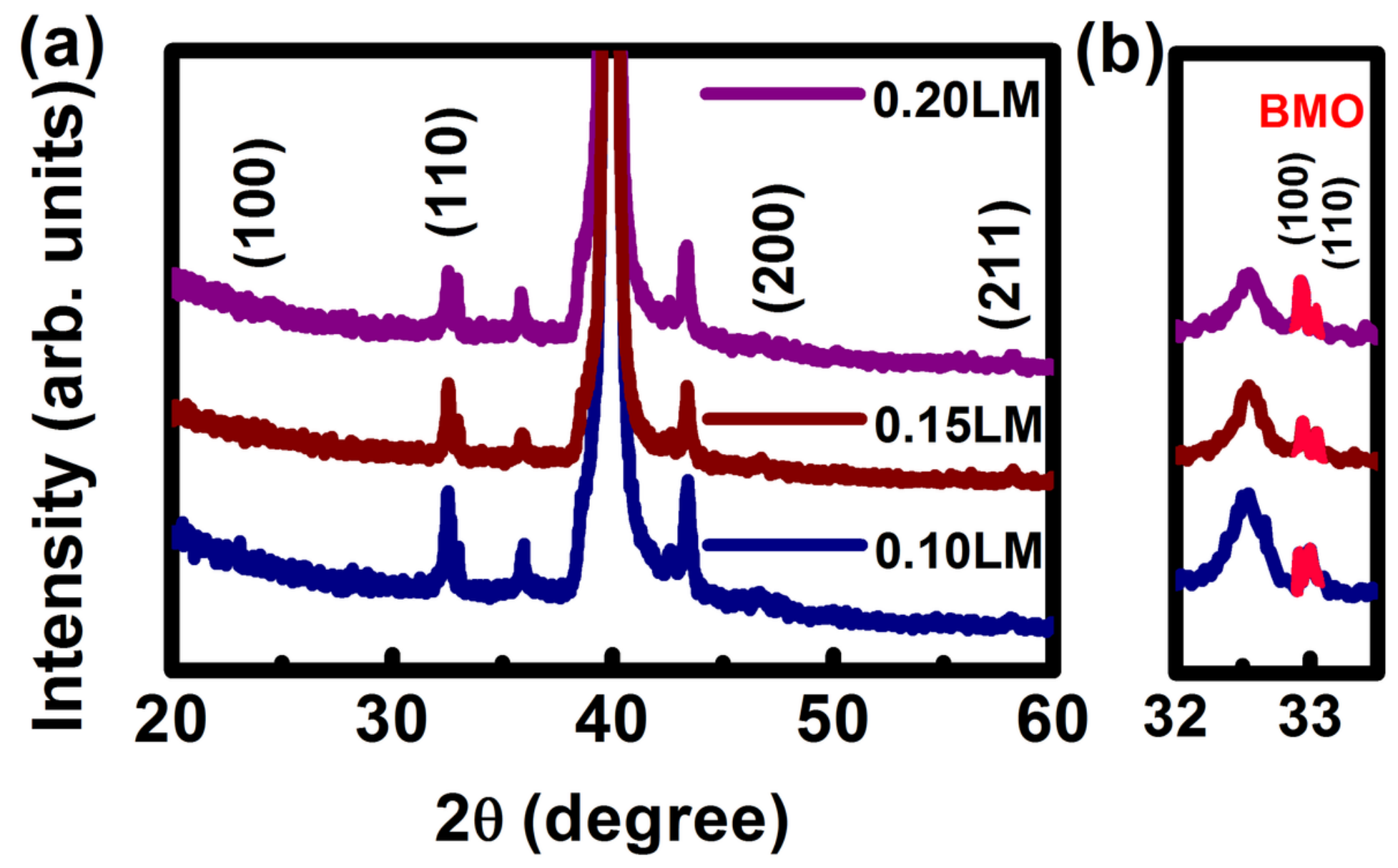

Figure 1

(a) XRD patterns of NBT-BM-LMO solid solution films, (b) detailed enlarged view of XRD patterns. 

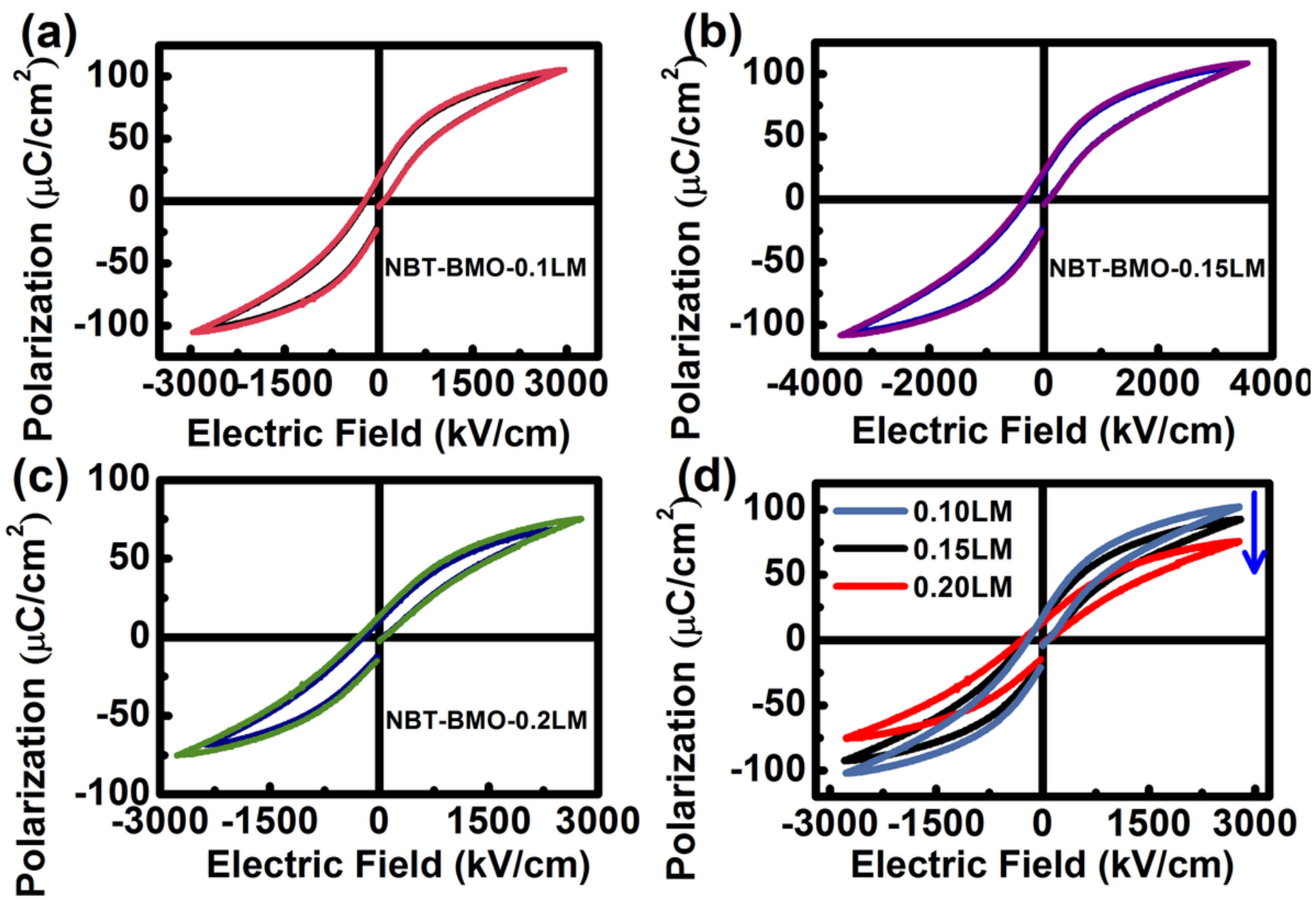

Figure 2

(a-c) P-E loops of NBT-BM-LMO solid solution with the LMO proportion of $0.1,0.15$ and 0.2 respectively, and (d) the change details of P-E loops under different LMO component at $2870 \mathrm{kV} / \mathrm{cm}$.
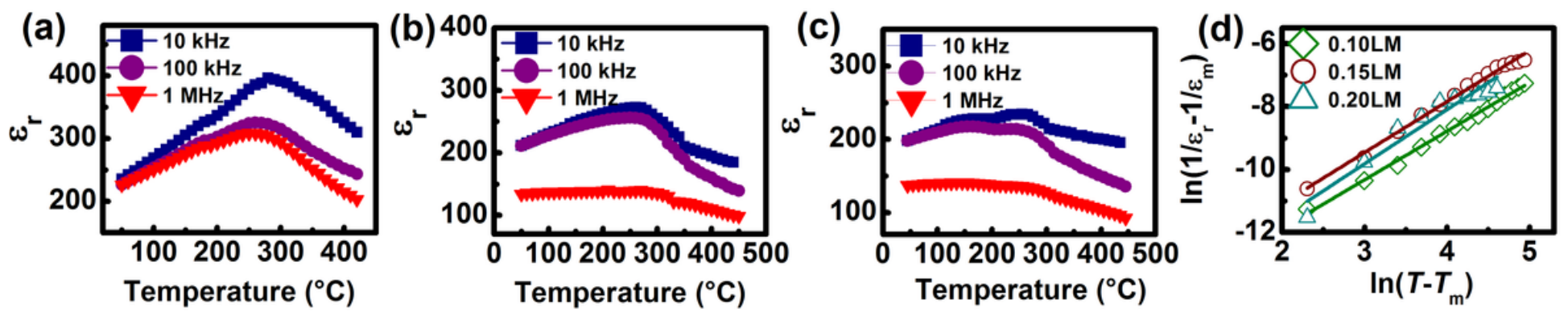

Figure 3

(a-c) the temperature-dependent dielectric constant of NBT-BM-LMO solid solution with the LMO proportion of $0.1,0.15$ and 0.2 respectively, (d) the dispersion coefficient $y$ for NBT-BM-0.1LMO, NBT-BM$0.15 \mathrm{LMO}$ and NBT-BM-0.2LMO films. 

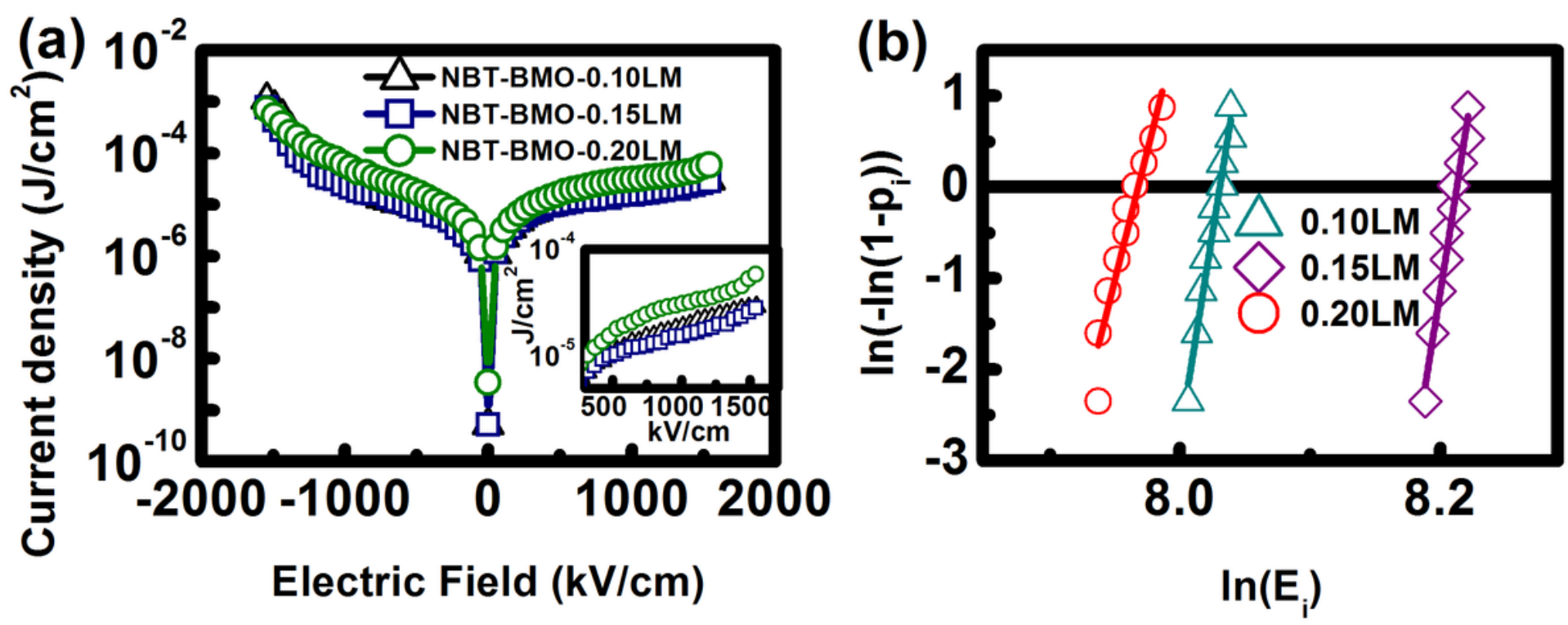

Figure 4

(a) The leakage current density of NBT-BM-LMO solid solution with LM proportion of $0.1,0.15$ and 0.2 respectively, (b) Weibull distribution of breakdown strength for NBT-BM-LMO solid solution with LM proportion of $0.1,0.15$ and 0.2 respectively.


Figure 5 
(a) the energy density and (b) efficiency of NBT-BM-LMO solid solution films under different components, (c) temperature-dependent energy storage performances, and (d) frequency-dependent energy storage performances. 